\title{
VARIAÇÃO NA MICROMEDIÇÃO DO CONSUMO DE ÁGUA NO FUNCIONAMENTO CORRETO E REVERSÍVEL DO HIDRÔMETRO
}

\section{MICRO MEASUREMENT VARIATION OF WATER CONSUMPTION IN CORRECT AND REVERSIBLE FUNCTIONING OF HYDROMETE}

\author{
Paulo Sérgio Scalize ${ }^{1}$ e Wellington Cyro de Almeida Leite ${ }^{2}$
}

Recebido em 11 de dezembro de 2012; recebido para revisão em 14 de dezembro de 2012; aceito em 26 de dezembro de 2012; disponível on-line em 28 de dezembro de 2012.

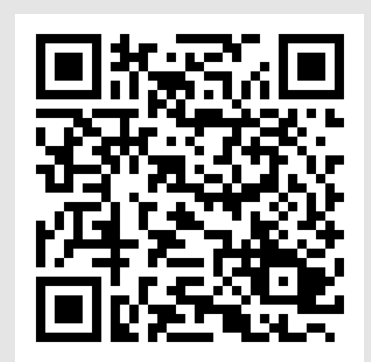

PALAVRAS CHAVES:

Hidrômetro;

Micromedição;

Perdas de água;

Consumo de água;

Medidor de água;

Reversível.

\section{KEYWORDS:}

Hydrometer;

Micro-measurement;

Water losses;

Water consumption;

Water measurement;

Reversible.

\begin{abstract}
RESUMO: Visando o combate às perdas aparentes, o parque de hidrômetros deve ser objeto de uma manutenção preventiva ativa, com avaliação criteriosa dos hidrômetros a serem substituídos. Uma das principais funções da substituição dos medidores é reduzir a submedição, registrando o consumo corretamente. No entanto, os medidores devem registrar o consumo no fluxo e no refluxo, fato esse que pode influenciar no volume registrado em um determinado período. Dessa forma, foram realizados estudos em bancada e em campo com o objetivo de verificar o desempenho de medidores velocimétricos e volumétricos novos de diferentes fabricantes. No estudo em bancada foram constatados erros variando de $-15,5 \%$ a $-100 \%$ no refluxo para os medidores velocimétricos e para os medidores volumétricos ensaiados os erros apresentados nas situações de estudo foram praticamente iguais, não influenciando no volume registrado pelo medidor. O estudo em campo evidenciou que o hidrômetro velocimétrico do fabricante A registrou, em 7 dias, $800 \mathrm{~L}$ a mais que o medidor volumétrico do fabricante $F$, evidenciando que o refluxo devido ao balanço de água na rede influencia diretamente no volume registrado pelo medidor velocimétrico, afetando a cobrança do consumo mensal do usuário do sistema de abastecimento de água. Visando resolver situações de refluxo, foram instaladas válvulas anti-retorno em dez ligações de água que apresentavam o problema de balanço de água na rede de distribuição. Nestes locais, os problemas foram resolvidos, além de ter sido observada uma pequena queda no volume registrado.
\end{abstract}

ABSTRACT: Aiming to combat the apparent losses, the hydrometer's park has to be object of preventive active maintenance, with careful evaluation of hydrometers to be replaced. One of the main functions of the measurer's replacement is reduce the sub-metering, registering the consumption accurately. However, the measurers should register the intake influx and reflux, that may influence the recorded volume in a determined period. Thus, benchtop and field studies were performed in order to verify the performance of volumetric and velocimetric new measurers, from different manufacturers. At benchtop study were observed ranging errors from $-15.5 \%$ to $-100 \%$ in reflux for velocimetric measurers, and to volumetric measurers tested, errors at study situations were virtually similar, don't influencing the volume recorded by measurer. The field study showed that velocimetric hydrometer from A's manufacturer registered, in 7 days, $800 \mathrm{~L}$ more than the volumetric meter from $\mathrm{F}^{\prime}$ 's manufacturer, indicating that reflux due to water balance in the network directly influences the volume registered by the velocimetric measurer, affecting the monthly charging of water's supply users. Aiming to solve reflux cases, backstop valves were installed in ten water connections that presented water balance problems at distribution network. In these places, the problems were solved, and was observed a small decrease in registered volume.

\footnotetext{
* Contato com os autores:

${ }^{1}$ e-mail : pscalize.ufg@gmail.com (P. S. Scalize)

Professor Dr. da Escola de Engenharia Civil da Universidade Federal de Goiás (EEC/UFG)

2 e-mail : wcyro@uol.com.br (W. C. de A. Leite)

Professora Dr. da Faculdade de Engenharia de Guaratinguetá - Universidade Estadual Paulista (UNESP) 


\section{INTRODUÇÃO}

Visando o combate às perdas aparentes, o parque de hidrômetros deve ser objeto de uma manutenção preventiva ativa, com avaliação criteriosa dos hidrômetros a serem substituídos.

Segundo estudos, autores indicam que a maioria dos hidrômetros instalados nos imóveis são de 3 $\mathrm{m}^{3} / \mathrm{h}$ ou menores. Garcia (2009) relata que na cidade de Campinas $70 \%$ das ligações prediais são providas de hidrômetros com vazão nominal $\left(Q_{n}\right)$ de $0,75 \mathrm{~m}^{3} / \mathrm{h}$, representando 175 mil hidrômetros.

Alguns problemas podem surgir em decorrência da utilização de medidores de água cada vez mais sensíveis. Estes problemas refletem-se na maioria das vezes em reclamações por parte dos usuários, sendo que as mais frequentes são o ponteiro do hidrômetro não para de girar; o ponteiro do hidrômetro gira sem estar havendo uso de água; o ponteiro do hidrômetro gira para frente e para trás; o hidrômetro está marcando a mais; o hidrômetro está com defeito; o hidrômetro está roubando para a empresa; o hidrômetro está registrando o ar; etc.

Para reduzir as reclamações por parte dos usuários muitas vezes são criados programas de conscientização, treinamentos dos atendentes e leituristas, campanhas educativas entre outras. Paul e Uliano (2006) concluíram em sua pesquisa que um treinamento realizado junto aos fiscais de rua $e$ atendentes do Serviço de Atendimento ao Cliente (SAC) foi importante para reduzir em $40 \%$ o número de solicitações (550 para 330 solicitações/ano). Scalize et al. (2007), relatam em seu trabalho que ações tais como criação de instrução de trabalho, folhetos explicativos para os usuários e treinamento dos atendentes foram suficientes para redução superior a $80 \%$ do número de solicitações de verificação do desempenho dos hidrômetros.

Conforme recomenda Tsutiya (2006), é conveniente que os medidores sejam retirados periodicamente, examinados, reajustados ou substituídos, para uma nova etapa de trabalho, evitando assim prejuízos à concessionária dos serviços de saneamento ou aos usuários.

A substituição por hidrômetros mais sensíveis agrava ainda mais as reclamações, pois a politica tarifária empregada na maioria dos órgãos responsáveis pelo abastecimento e cobrança pelo uso da água no Brasil, utilizam a cobrança por faixa, com efeito "cascata". Portanto, o consumidor que gasta mais entra em faixas superiores onde o valor do volume utilizado é maior.
Outra situação comumente enfrentada é a reclamação de ar na rede onde pesquisas indicam que os bloqueadores de ar podem não ter beneficio para o consumo e ser prejudicial ao sistema de abastecimento conforme pesquisa desenvolvida por LOPES et al (2011), e dizem ainda que quanto menor o consumo do usuário, maior poderá ser a influência do ar no valor final da conta mensal.

Desta forma, torna-se fundamental o esclarecimento à população, bem como estudos aprofundados sobre a qualidade de um medidor de água e seu correto funcionamento.

Neste trabalho foi dada ênfase às reclamações de que "o ponteiro do medidor gira para frente e para trás". Este acontecimento é ocasionado pelas variações de pressão entre os ramais e a rede de distribuição, sendo um fenômeno conhecido como "Balanço de Rede", observado principalmente em ramais com desmembramento, ou seja, em ramais onde é feita uma derivação para abastecer outro imóvel.

O "Balanço de Rede" ocorre devido às variações de pressão decorrentes da vazão, causadas por alguma alteração, voluntária ou involuntária, imposta ao fluxo de líquidos no interior de condutos. Isto quer dizer que ocorre quando se aumenta ou diminui a vazão, porém de uma maneira suficientemente rápida para que as forças elásticas do líquido e do conduto sejam mobilizadas, dando origem a ondas de pressão.

Segundo a Portaria 246 do INMETRO, de 17 de outubro de 2000 o hidrômetro deve permitir o funcionamento reversível conforme item 3.6:

"3.6 Funcionamento reversível do hidrômetro.

3.6.1 O hidrômetro deve permitir o funcionamento reversível por um período de seis minutos, na vazão nominal, registrando indicações no sentido inverso, sem se danificar e sem alterar suas qualidades metrológicas, quando novamente submetido ao sentido normal do fluxo."

Sendo um critério para aprovação do modelo conforme letra $\mathbf{b}$ do item 6.4.1:

"6.4.1 Os ensaios para aprovação de modelo de hidrômetros são os abaixo indicados:

b) verificação de funcionamento inverso;"

No entanto, a Portaria 246/00 não comenta sobre a margem de erro permitida. Desta forma, o simples fato do medidor registrar um volume inverso, mesmo com altas porcentagens de erros, está de acordo com a legislação vigente. 
Segundo Coelho (1996) o princípio dos medidores velocimétricos consiste na obtenção do volume de água que atravessa um aparelho, contando o número de revoluções da turbina. A medição é obtida de forma indireta pela correlação existente entre o número de revoluções da turbina e o volume de água que atravessa o aparelho. Em resumo, baseia-se no fato de que para um mesmo diâmetro e um mesmo orifício, o número de revoluções " $N$ " será diretamente proporcional a velocidade.

Assim sendo, o volume de água que atravessa o medidor é obtido pela Equação 1.

$$
\mathbf{V}=\mathbf{K} \times \mathbf{N}
$$

Onde:

$\mathbf{V}=$ volume de água que atravessa o medidor;

$\mathbf{K}=$ constante que depende da forma e superfície do orifício e do diâmetro da turbina;

$\mathbf{N}=$ número de revoluções da turbina.

Os medidores volumétricos, segundo Coelho (1996), aplicam o princípio de uma câmara de volume conhecido, de um elemento móvel, situado dentro dela, que adquire ao passar a água um movimento cíclico, onde continuamente se enche e esvazia a câmara. Desta forma o volume é registrado.

Diante do exposto, serão discutidas, neste trabalho possíveis causas que provocam medições de vazões, acima do consumo efetivo, mesmo em medidores aprovados pelo INMETRO (Instituto Nacional de Metrologia, Normalização e Qualidade Industrial) e suas prováveis soluções.

Dessa forma, o presente trabalho tem como objetivo verificar o desempenho de medidores de água, velocimétrico e volumétrico, quando submetidos a condições de refluxo, ou seja, no sentido inverso.

\section{METODOLOGIA}

Este trabalho foi realizado em três fases, descritas a seguir:

\subsection{ENSAIOS EM LABORATÓRIO}

Nesta fase foram utilizados medidores velocimétricos, classe $B$, com $Q_{n}=0,75 \mathrm{~m}^{3} / \mathrm{h}$, de cinco fabricantes diferentes (marca $A, B, C, D$ e E) e medidor volumétrico, classe $C$, com $Q_{n}=1,50 \mathrm{~m}^{3} / \mathrm{h}$, de dois fabricantes diferentes (marca F e G).

Os medidores foram submetidos a ensaios para verificação do desempenho de medição (erros de medição), em bancadas próprias e devidamente inspecionadas pelo IPEM (Instituto de Peso e Medidas), órgão credenciado pelo INMETRO. Os ensaios foram realizados seguindo as normas da Portaria 246/00 para a vazão mínima $\left(Q_{\min }\right)$, transição $\left(Q_{t}\right)$ e nominal $\left(Q_{n}\right)$. Além destas, foi realizado ensaio com a vazão determinada para início de funcionamento de cada modelo. As vazões de ensaios estão descritas na Tabela 1, e foram realizadas de duas formas, sendo uma com o fluxo de água no sentido correto (fluxo) e outra com o fluxo de água invertido (refluxo).

Tabela 1: Vazões utilizadas nos ensaios para verificação dos erros dos medidores.

\begin{tabular}{|c|c|c|c|c|}
\hline \multirow{3}{*}{ Vazões de ensaios } & \multicolumn{4}{|c|}{ Vazões de ensaios $\left(\mathrm{m}^{3} / \mathrm{h}\right)$} \\
\hline & \multicolumn{2}{|c|}{$\begin{array}{l}\text { Medidor Velocimétrico Classe B } \\
\qquad Q_{n}=0,75 \mathrm{~m}^{3} / \mathrm{h}\end{array}$} & \multicolumn{2}{|c|}{$\begin{array}{l}\text { Medidor Volumétrico Classe } C \\
\qquad Q_{n}=1,5 \mathrm{~m}^{3} / \mathrm{h}\end{array}$} \\
\hline & Fluxo & Refluxo & Fluxo & Refluxo \\
\hline $\mathbf{Q}_{\text {início de funcionamento }}$ & 0,008 & 0,008 & 0,002 & 0,002 \\
\hline $\mathbf{Q}_{\min }$ & 0,015 & 0,015 & 0,015 & 0,015 \\
\hline$Q_{t}$ & 0,060 & 0,060 & 0,0225 & 0,0225 \\
\hline $\mathbf{Q}_{\mathrm{n}}$ & 0,750 & 0,750 & 1,500 & 1,500 \\
\hline
\end{tabular}




\subsection{ENSAIOS EM CAMPO}

Além dos testes em bancada, foi realizado um ensaio de campo em um imóvel onde existe balanço de água na rede de distribuição. Neste local, foi instalado um conjunto de medidores (Figura 1), sendo composto por um medidor velocimétrico do fabricante $A$ (que apresentou maior margem de erro na medição em refluxo), classe $B$, com $Q_{n}=0,75 \mathrm{~m}^{3} / \mathrm{h}$, e um medidor volumétrico, classe $C$, com $Q_{n}=1,50 \mathrm{~m}^{3} / \mathrm{h}$, com saída pulsada e um registrador de consumo (logger). Este equipamento emite um pulso a cada litro, sendo coletadas as leituras para traçar um gráfico da variação do consumo ao longo do período estudado.

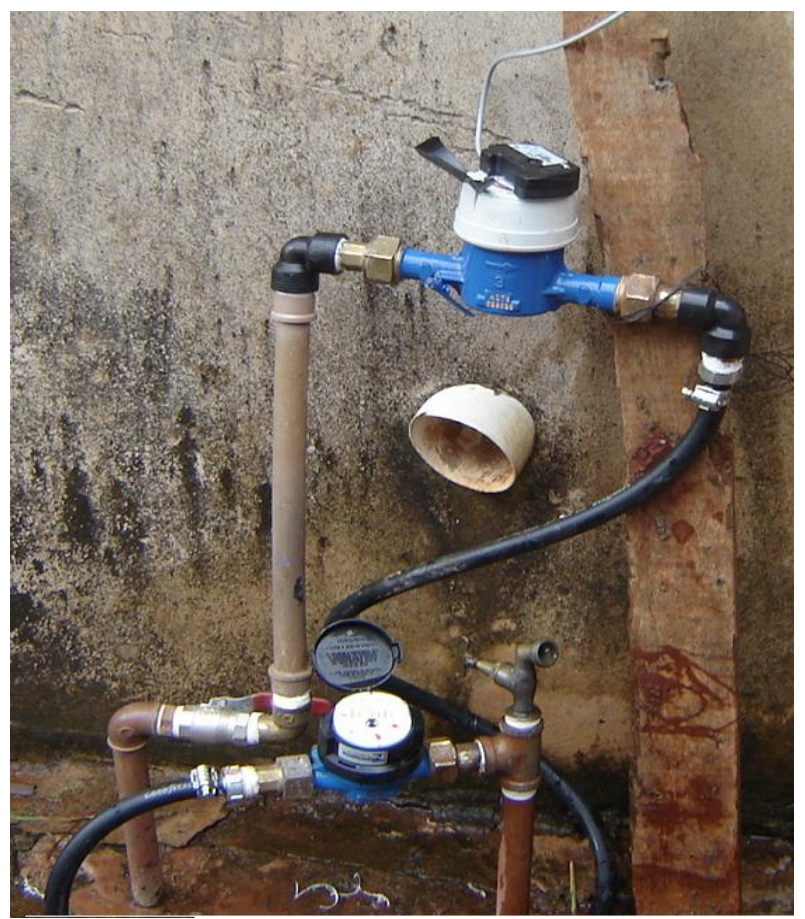

Figura 1: Conjunto de medidores instalados em série, composto por um medidor velocimétrico e por um volumétrico com registrador de volume.

\subsection{INSTALAÇÕES DE VÁLVULOAS ANTI-RETORNO}

Foram selecionados 10 imóveis para a instalação de válvulas anti-retorno (Figura 2) com a finalidade de impedir o refluxo. Desta forma, fica impossível o ponteiro do medidor girar para trás (que poderia ocorrer caso houvesse defeito na peça), ou seja, grande parte das reclamações dos consumidores foi resolvida.

O modelo de válvula escolhido foi de um fabricante que inseriu um dispositivo plástico antiretorno dentro de um tubete, o que facilita sua instalação no cavalete, pois substitui o tubete tradicional.

A instalação de válvula anti-retorno impede, além do ponteiro do medidor girar para trás, a contaminação da rede por sifonamento e refluxo de substâncias prejudiciais, conforme pode ser observado no esquema da Figura 3.

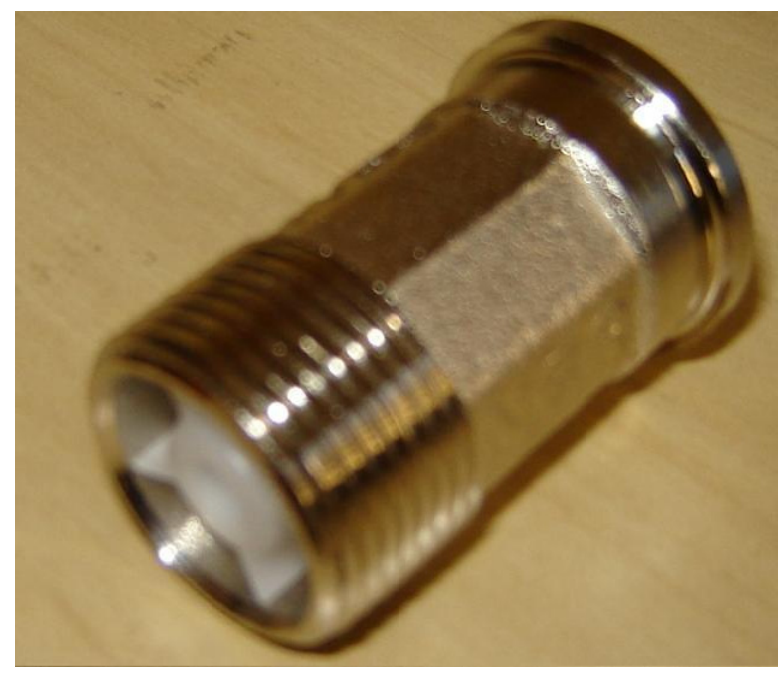

Figura 2: Válvula anti-retorno utilizada nas instalações para impedir o refluxo. Observar no detalhe o dispositivo em plástico que é inserido no interior do tubete.

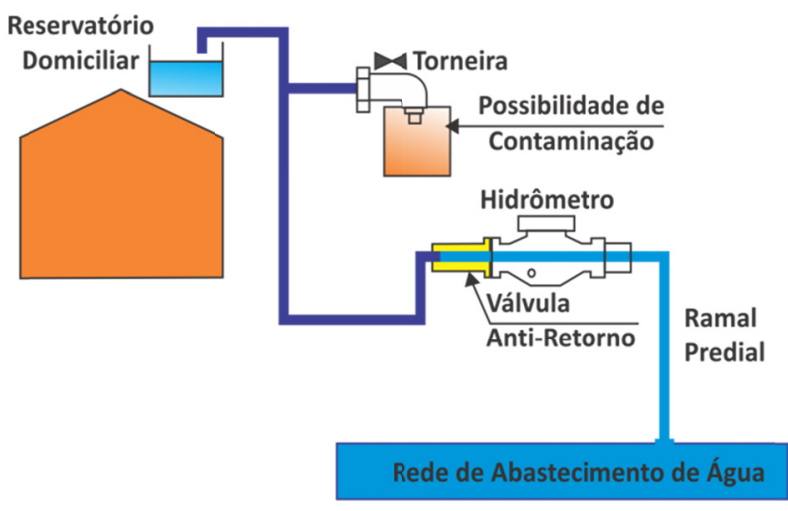

Figura 3: Esquema de instalação de uma válvula antiretorno. Observar que, caso houvesse um refluxo poderia ocorrer contaminação pelo líquido sifonado na torneira.

\section{RESULTADOS E DISCUSSÕES}

\subsection{ENSAIOS EM LABORATÓRIO}

Os resultados obtidos nos ensaios em laboratório, visando a verificação dos erros dos medidores, estão inseridos na Tabela 2. Para os medidores velocimétricos que foram instalados para medir o refluxo, observou-se uma grande margem de erro na medição para o fabricante $A$ e $E$, superior a $64 \%$, diminuindo para os fabricantes $B, C$ e $D$, sendo que, com exceção do medidor $\mathrm{C}$, os demais medidores apresentaram erro superior a - 51\% em qualquer vazão.

Os ensaios com os diferentes medidores velocimétricos deixaram claro que os erros apresentados 
são influenciados pelas condições mecânicas do medidor, característico de cada marca, variando de $15,5 \%$ a - 100\%. Isto ocorre devido ao diâmetro de entrada do fluxo de água, quando no sentido correto e no refluxo de cada fabricante. $O$ diâmetro interfere na velocidade do fluxo sobre a turbina, fazendo com que ocorra maior ou menor erro em função da diferença dos diâmetros de entrada e saída do medidor. Isto pode ser explicado utilizando a Equação da Continuidade $(\mathrm{Q}=\mathrm{V} \mathrm{x}$ A), sendo que para uma mesma vazão utilizando um diâmetro maior, a área será maior e consequentemente a velocidade será menor, fazendo com que o medidor registre o volume com maior erro, pois ele é velocimétrico.

Na Figura 4 pode ser observada a variação dos diâmetros de entrada e saída dos medidores velocimétricos. Foi verificado que os maiores erros no refluxo acontecem com os medidores dos fabricantes $A$ e $B$, que apresentam uma grande diferença entre os seus diâmetros de entrada e saída. Este fato não ocorre com o

saida. Este fato não oco medidor do fabricante $C$, onde os diâmetros de entrada e saída são quase iguais, refletindo em um erro bem menor no refluxo.

Em contrapartida o medidor volumétrico do fabricante $F$ e $G$, apresentaram erros próximos tanto no fluxo como no refluxo. Vale destacar que o medidor do fabricante $F$ apresentou os mesmos erros para as vazões mínima $(+0,5 \%)$, de transição $(+2,0 \%)$ e máxima $(+0,9 \%)$ tanto no fluxo como no refluxo. Desta forma fica evidenciando que os medidores volumétricos registrariam o real consumo do usuário, sem distorcer o seu consumo para mais ou para menos.

Os hidrômetros velocimétricos instalados na cidade de estudo nos anos de 2006 e 2007 foram, respectivamente, dos fabricantes $A$ e C. Neste período, as reclamações por parte dos usuários com relação a oscilação do ponteiro do medidor recaiu apenas sobre os do fabricante $A$, evidenciando que sua sensibilidade em vazões menores é inferior que a do fabricante $C$. Desta forma, a sub-medição para os medidores do fabricante $A$ é menor que a do fabricante $C$.
FABRICANTE A

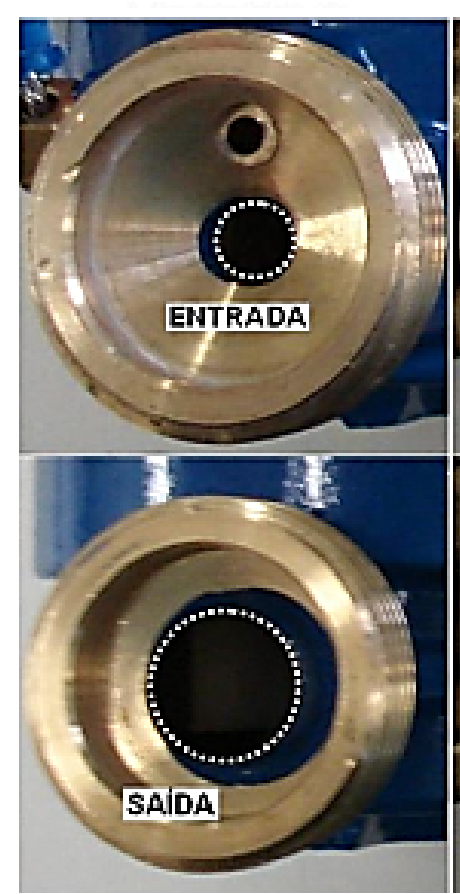

Figura 4: Comparação entre os diâmetros dos orifícios de entrada e saída dos medidores velocimétricos dos fabricantes $\mathrm{A}, \mathrm{B}$ e $\mathrm{C}$.

\section{FABRICANTE B}
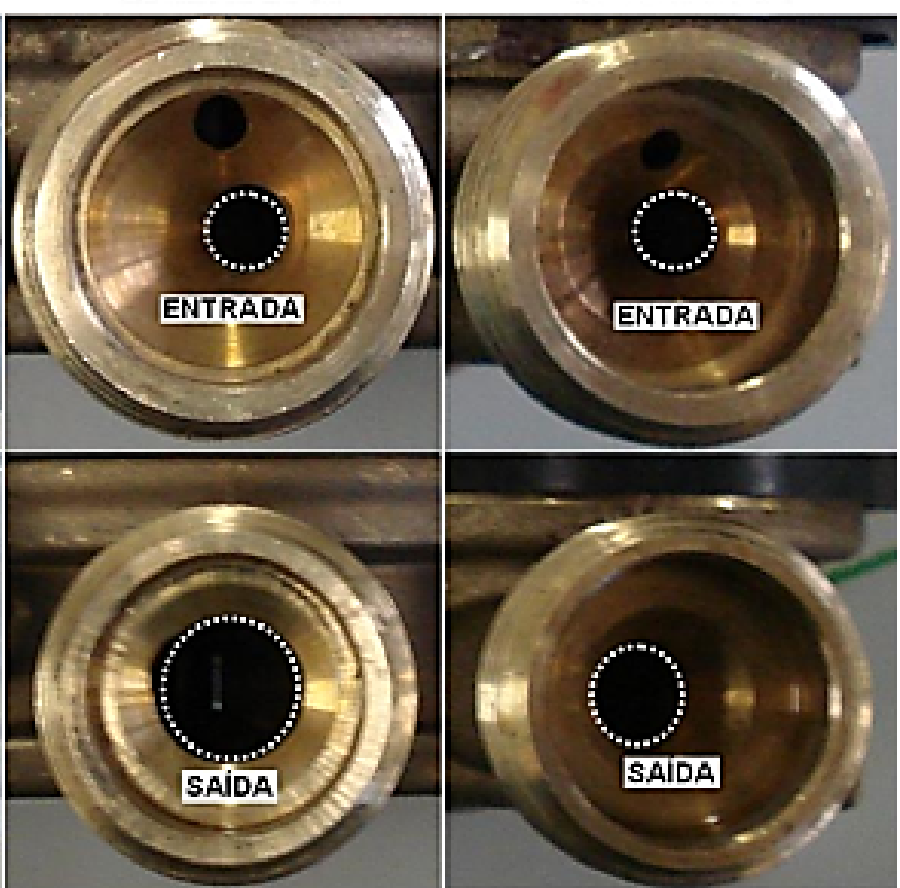

\section{FABRICANTE C}


Tabela 2: Resultados dos ensaios para verificação do desempenho de cada medidor, no sentido correto (fluxo) e no refluxo.

Erros dos medidores ensaiados (\%)

\begin{tabular}{|c|c|c|c|c|c|c|c|c|c|c|c|c|c|c|}
\hline \multirow{3}{*}{$\begin{array}{l}\text { Vazões } \\
\text { de } \\
\text { ensaios }\end{array}$} & \multicolumn{10}{|c|}{$\begin{array}{l}\text { Medidor Velocimétrico Classe B } \\
\qquad Q_{n}=0,75 \mathrm{~m}^{3} / \mathrm{h}\end{array}$} & \multicolumn{4}{|c|}{$\begin{array}{l}\text { Medidor Volumétrico Classe } \\
\qquad C \\
\qquad Q_{n}=1,5 \mathrm{~m}^{3} / \mathrm{h}\end{array}$} \\
\hline & \multicolumn{2}{|c|}{ Marca A } & \multicolumn{2}{|c|}{ Marca B } & \multicolumn{2}{|c|}{ Marca C } & \multicolumn{2}{|c|}{ Marca D } & \multicolumn{2}{|c|}{ Marca E } & \multicolumn{2}{|c|}{ Marca F } & \multicolumn{2}{|c|}{ Marca G } \\
\hline & Fluxo & Refluxo & Fluxo & Refluxo & Fluxo & Refluxo & Fluxo & Refluxo & Fluxo & Refluxo & Fluxo & Refluxo & Fluxo & Refluxo \\
\hline $\begin{array}{l}\mathbf{Q}_{\text {início de }} \\
\text { funcionamento }\end{array}$ & $-8,4$ & $-100,0$ & $-14,8$ & $-99,6$ & $-15,0$ & $-43,5$ & $-9,2$ & $-100,0$ & $-100,0$ & $-100,0$ & $-2,0$ & $-4,0$ & $-9,0$ & $-13,5$ \\
\hline $\mathbf{Q}_{\min }$ & $+1,2$ & $-75,0$ & $-1,8$ & $-61,4$ & 0,0 & $-18,5$ & $+2,6$ & $-56,6$ & $-0,5$ & $-90,5$ & $+0,5$ & $+0,5$ & $-0,5$ & $-1,5$ \\
\hline$Q_{t}$ & $+1,0$ & $-67,2$ & $+0,8$ & $-51,0$ & 0,0 & $-15,5$ & $+1,0$ & $-51,0$ & $+0,5$ & $-77,0$ & $+2,0$ & $+2,0$ & $+0,5$ & 0,0 \\
\hline $\mathbf{Q}_{\text {máx }}$ & $-0,6$ & $-69,4$ & $+0,8$ & $-51,5$ & $-0,5$ & $-15,9$ & 0,0 & $-51,6$ & $-1,6$ & $-64,8$ & $+0,9$ & $+0,9$ & $+1,1$ & $+1,2$ \\
\hline
\end{tabular}

\subsection{ESTUDOS EM CAMPO}

O estudo em campo evidenciou que o hidrômetro velocimétrico do fabricante $A$ registrou, em sete dias, $800 \mathrm{~L}$ a mais que o medidor volumétrico do fabricante $F$, evidenciando que o balanço de água na rede influencia diretamente no volume registrado pelo medidor velocimétrico, afetando a cobrança do consumo mensal do usuário do sistema de água. Neste caso o volume registrado, erroneamente, poderá atingir, aproximadamente, $3,5 \mathrm{~m}^{3}$ de água num período de 30 dias. Considerando que o consumo médio residencial da cidade de estudo é da ordem de $20 \mathrm{~m}^{3} / \mathrm{mês}$, o volume registrado representa $17,5 \%$ a mais.

Certamente, se fosse utilizado o medidor do fabricante $F$ ou $G$ o aumento do volume registrado erroneamente seria menor, ou ainda não existiria.

\subsection{INSTALAÇÃO DE VÁLVULAS ANTI-RETORNO}

Foram instaladas válvulas anti-retorno em dez ligações de água que apresentavam o problema de balanço de água na rede de distribuição, sendo que, o ponteiro do hidrômetro girava para frente e para trás. Vale ressaltar que, as voltas do ponteiro para frente são maiores que no refluxo, ou seja, três ou quatro voltas para frente e apenas uma para trás. Nestes locais, os problemas foram resolvidos e os consumidores ficaram satisfeitos, além de observar uma pequena queda no volume registrado.

\section{CONCLUSÃO}

O presente trabalho permitiu concluir que:
- o balanço de água na rede de distribuição exerce influência sobre o volume de água medido, sendo que as reclamações dos consumidores são procedentes;

- dependendo da qualidade do medidor utilizado e da maior intensidade do balanço de água na rede de distribuição, o consumo registrado indevidamente pode ser muito expressivo;

- em locais onde existe o balanço de água na rede de distribuição é necessária a substituição de medidores velocimétricos por medidores volumétricos ou, caso queira manter os medidores velocimétricos, torna-se imprescindível a aquisição de medidores com a mesma sensibilidade de medição no fluxo e no refluxo, ou ainda, a instalação de uma válvula antiretorno, o que evitaria o refluxo;

- medidores velocimétricos com maior sensibilidade para registrar vazões menores apresentam maiores índices de reclamações, no entanto, registram com melhor desempenho;

- a utilização de medidor volumétrico resolveria o problema de registrar consumo indevidamente. No entanto, as reclamações por parte dos usuários continuariam devido ao fato do ponteiro do medidor continuar girando para frente e para trás.

\section{REFERÊNCIAS BIBLIOGRÁFICAS}

COELHO, A. C.. Medição de Água, Política e Prática. Recife: Editora Comunicarte, 1996, 358 p.

GARCIA, M.A.. Análise do desempenho em campo de hidrômetros Qn $\mathbf{0 , 7 5} \mathrm{m}^{\mathbf{3}} / \mathbf{h}$. In: Exposição de Experiências Municipais em Saneamento, 13a , Gramado - RS. Anais eletrônicos, Brasília: ASSEMAE. 2009, 10p. 
INSTITUTO NACIONAL DE METROLOGIA, NORMALIZAÇÃO E QUALIDADE INDUSTRIAL (2000). INMETRO, Portaria № 246 de 17 de outubro de 2000.

LOPES, N.P; LARA, M.; LIBÂNIO, M.. Quantificação em escala de bancada do volume de ar em ligações prediais de água. Revista Engenharia Sanitária e Ambiental, Rio de Janeiro, v. 16, n.4, out/dez, p. 343-352, 2011.

PAUL, V.; ULIANO, A. (2006). Exame metrológico e análise de desempenho de hidrômetros, buscando excelência e qualidade no atendimento ao cliente. In: Exposição de Experiências Municipais em Saneamento, 10a , Joinville - SC. Anais eletrônicos, Brasília: ASSEMAE. 2006, 8p.

SCALIZE, P.S.; PERONI, J.C.A.. Ações visando a minimização das solicitações de verificações do desempenho dos hidrômetros por parte dos consumidores. In: Exposição de Experiências Municipais em Saneamento, 11ạ, Guarulhos SP. Anais eletrônicos, Brasília: ASSEMAE. 2007, 9p.

Tsutiya, M.T.; Abastecimento de Água $-4^{\mathrm{a}}$ Ed. - São Paulo Departamento de Engenharia Hidráulica e Sanitária da Escola Politécnica da Universidade de São Paulo - USP, 2006, $643 \mathrm{p}$. 